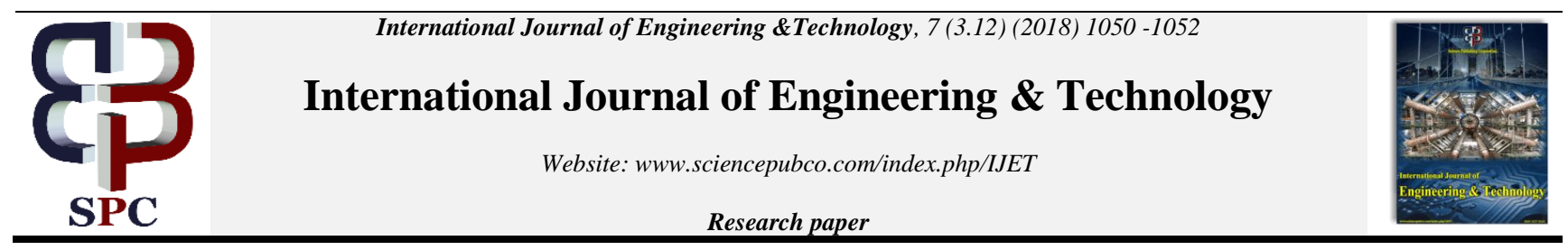

\title{
An Efficient Spectral Spatial Classification for Hyper Spectral Images
}

\author{
G. Shanmugapriya* \\ Professor, Department of ECE, CMR Institute of Technology, Hyderabad, Telangana \\ *Corresponding Author Email: spriyagsn@yahoo.com
}

\begin{abstract}
An expanded random walker comprises of two primary advances ghostly spatial order strategy for hyper Ghastly pictures. To begin with go to pixel astute order by utilizing bolster vector machine (SVM) which is arrangement likelihood maps for a hyper unearthly picture. Probabilities of hyper phantom Pixel have a place with various classes. The second approach is getting pixel shrewd likelihood maps are upgraded broadened arbitrary walker calculation. Pixel astute measurements data by SVM classifier, spatial relationship between neighboring pixels displayed through weight of diagram edges preparation and test tests demonstrated irregular walkers. These 3 components utilizing for the class of validating pixel are resolved. So, these three elements considered in ERW. The proposed technique demonstrates great order performs for three generally utilized genuine hyper otherworldly informational collections even the quantity of preparing tests is moderately little.
\end{abstract}

Keywords: Extended random walkers; hyper spectral images; optimization; spectral-spatial classification; $k$-means.

\section{Introduction}

Hyper otherworldly sensors go get a gander at objects utilizing a tremendous part of the electro attractive range, this pictures like other ghastly imaging, gathers and procedures data from over the electro attractive range. Hyper unearthly pictures are given abnormal state understanding remotely detected pictures [1]. The exceptional quality of informational indexes of hyper ghastly is picturesque grouping in the phantom hyperspace still has numerous settled issues. High dimensionality of informational indexes includes huge wonder in grouping. This wonder gives number of preparing tests are adjusted, the arrangement precision might be diminishing for some managed characterization strategies as the information dimensionality increment. So, manage this trouble, to construct a few arrangements they are 1. Feature extraction

a. Principal component analysis

b. Independent component analysis

The related activities high dimensional information into low measurement information into a low dimensional element space while safeguarding the discriminative data of various classes bolster vector machines, multi nominol strategic relapse of discriminative learning approaches learn class appropriation in high dimensionality in include space. So these techniques are troubles caused by high dimensionality[2]. The utilization of spatial data of the information is to enhance the arrangement precision to develop the phantom spatial hyper ghastly grouping techniques rather than huge wonder. Otherworldly spatial strategies, for example, nonlocal joint communitarian, spatial bit based techniques, probabilistic demonstrating based strategies. Be that as it may, in this proposed strategy is probabilistic demonstrating[3] based ghostly spatial grouping technique. ERW calculation is utilized for other worldly spatial order of hyper ghastly pictures. In this algorithm have two following steps:
1. pixel shrewd characterization by utilizing SVM is utilized to acquire as set of likelihood maps which measures likelihood that pixel has a place with class.

2. ERW is utilized for the ascertain an arrangement of streamlined likelihood all together for class of every pixel to decide considering greatest likelihood.

ERW improvement implies pixel shrewd otherworldly data, the spatial data between adjoining pixels and separation between the preparation and test pixels are joined. To get characterization result with high exactness than consequences of SVM, significantly number of preparing tests is little [4].

\section{ERW Algorithm}

Each edge eij, jth pixels $w(e i j)=e-\beta(v i-v j) 2$ where $\beta$ is free parameter.

The RM algorithm consists of following steps based on the weighted graphs.

1. set the marked pixels $V M$, each pixel vi belongs to $L=\{1,2$,----$---\mathrm{N}\}$

2. RW algorithm computes the probabilities Pin with the marked pixels

E spatialn $(\mathrm{pn})=\mathrm{p} \mathrm{nT} \mathrm{L} \mathrm{p} \mathrm{n}$

$\mathrm{Lij}=\mathrm{d} \mathrm{i} \quad$ if $\quad \mathrm{i}=\mathrm{j}$

$-w(e i j) \quad$ if $i, j$ together pixels

0

else

$\mathrm{di}=\sum \mathrm{w}($ eij) is degree of the ith pixel vi. i.e; ERW another spatial energy function is

$\operatorname{Easpatial}(\mathrm{pn})=\sum \mathrm{qN}=1, \mathrm{q} \neq 1 \mathrm{pqT} \wedge \mathrm{q} \mathrm{pq}+(\mathrm{pn}-1) \mathrm{T} \wedge \mathrm{n}(\mathrm{pn}-1)$ 
$\gamma$ is free parameter

$\operatorname{En}(\mathrm{pn})=\operatorname{Enspatial}(\mathrm{pn})+\gamma \operatorname{Enaspatial}(\mathrm{pn})$

\section{Proposed Method}

In this proposed approach have two main steps

A. Probability Estimation (PE) with SVM

B. Probability Optimization (PO) with ERW

\section{A. PE- SVM}

$\mathrm{L}=\{1,2$, $-, N\}, n^{\in} L$ refers to the $n$th class. $S=\{1,2,----, I\}$ be the set pixels of the hyper spectral image, $x=(x 1, x 2$, ----xi) ${ }^{\in}$ Rd*I hyper spectral

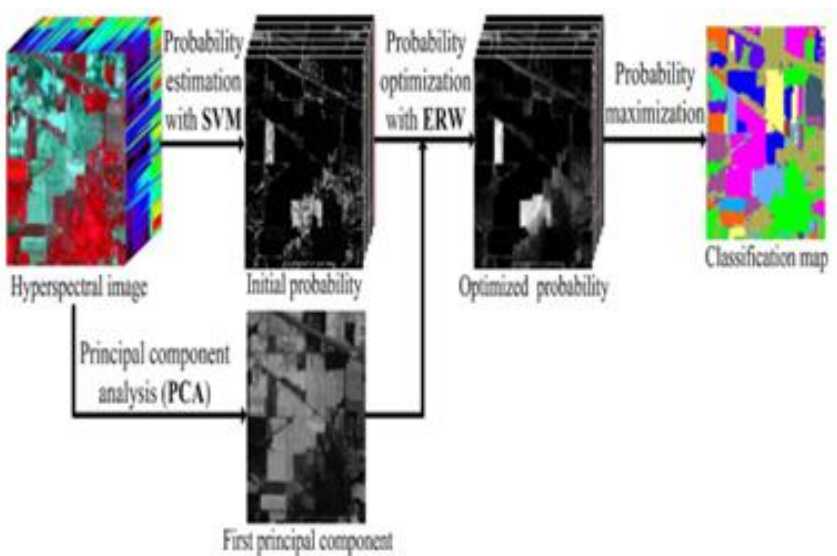

Fig. 1: Representation of projected ERW created spectral - spatial classification system

$\mathrm{TT}=\left\{(\mathrm{X} 1, \mathrm{X} 2-\mathrm{-}-\mathrm{XT})^{\in}\left(\mathrm{Rd}^{*} \mathrm{~L}\right) \mathrm{T}\right.$ of SVM, to get the probability maps r=(r1,r2---------rn).

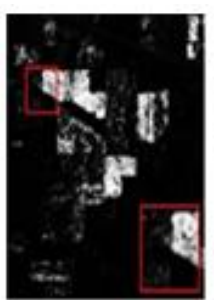

(a)

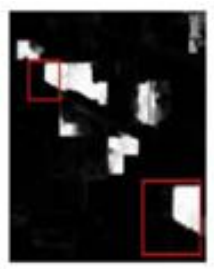

(e)

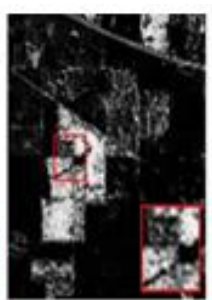

(b)

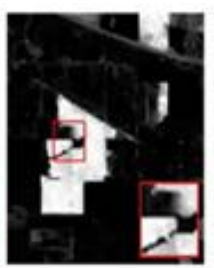

(f)

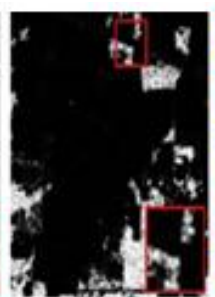

(c)

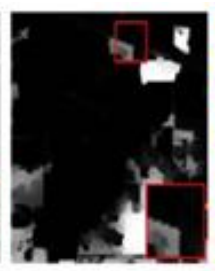

(g)

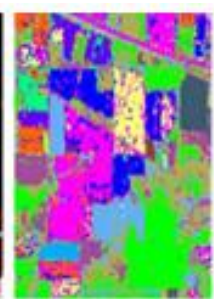

(d)

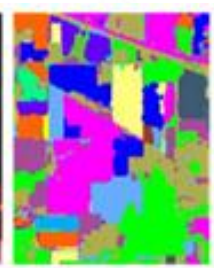

(h)
Fig. 2: (a)- (c) 3 introductory likelihood maps assessed by SVM comparing to various sorts of yields in the scene. (d) Classification outline SVM. (e) - (g) Optimized likelihood maps ERW. (h) Organization outline by future ERW-founded order technique. (a) r1. (b) r5. (c) r14. (d) SVM. (e) p1. (f) p5. (g) p14. (h) ERW.

In the above figure 2 from a to c noise is occurred. Since spatial information not considered and maximum probability of map also noise is occurring.

\section{B. Probability Optimization with ERW}

In this progression, ERW is taken to ensure the likelihood enhancement. This strategy comprises two stages. PCA is utilized for unearthly dimensional of the hyper phantom picture is lessened to build weighted chart $\mathrm{G}=(\mathrm{V}, \mathrm{E})$ The improved probabilities are acquired by minimization the vitality work in (4) in which the primary term is spatial relationship among contiguous pixels and second term coordinates the initial likelihood maps estimated by SVM Markov irregular strategy in the advancement procedure here every Pixel is utilizing the same weight coefficients for its spatial term pixels on a similar comparable names will have forces. The ERW strategy improves the precision in homogenous districts as well as upgrade probabilities adjust well to genuine protest limits. The above fig. 2 from e to g upgraded i.e, blunders areas properly expelled and improved probabilities are adjusted well to genuine boundaries.2(h) fig is relating border outline. The correlation between the $2(\mathrm{~d})$ and $2(\mathrm{~h})$ is more precisely after advancement.

\section{Experiments}

\section{A. Datasets}

Evaluated the execution the diverse characterization.

1. Universities pavia dataset: This dataset of picture of pictures estimate is $610 * 340$ pixels. The spatial determination is $1.3 \mathrm{~m}$ for every pixel. Before order 12 groups are taken because of commotion.

2. Salian Informational Indexes: This dataset of picture contains 224 groups of size $512 * 217.20$ water assimilation groups were disposed of before characterization. 3.Indian pines dataset This sort of pictures $145 * 145$ pixels. The spatial determination is $20 \mathrm{~m}$ for every pixel.12 channels are taken before grouping.

\section{B. Parameters Setting}

Proposed technique is contracted and the other ghastly spatial arrangement strategy. For example, SVM, EMP, LORSALMLL, MPM-LBP, EPF, IFRF. SVM parameters chose utilizing five overlaps cross validation. The EMP is developed utilizing initial four essential parts and a roundabout basic component with step estimate augmentation of two. LORSAL-MILL, MPM-LBP, EPF, IFRF strategies are actualized default parameters. In this proposed ERW technique parameters $\beta$ adv assessed by objective and visual examination. For underneath fig 3 University of pavia give got by SVM technique and the proposed strategy with various estimation of $\beta$ and general precision and normal exactness of proposed strategy if $\beta$ and values fluctuation can be seen in beneath fig 4 .
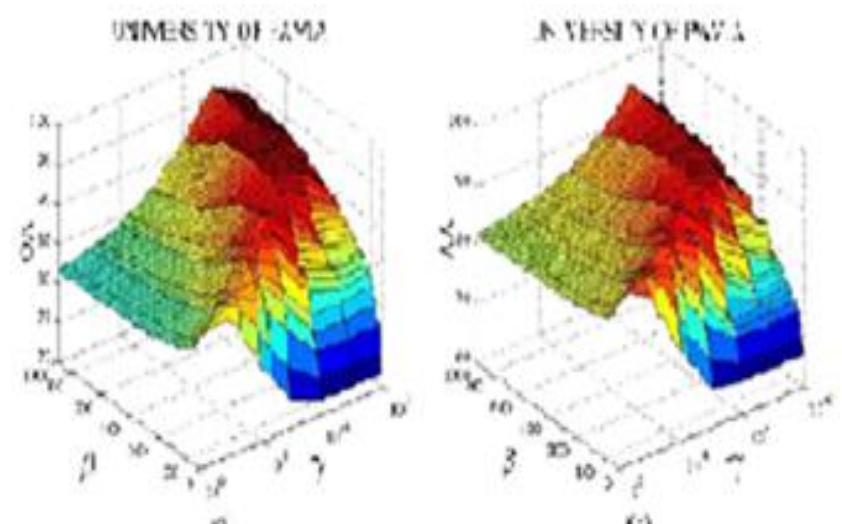

(i)

Fig. 4: Parameters on classification accuracies with different values of $\beta$ and $\gamma$ (University of Pavia data set). (a) OA and (b) AA 


\section{University of Pavia Data Set}

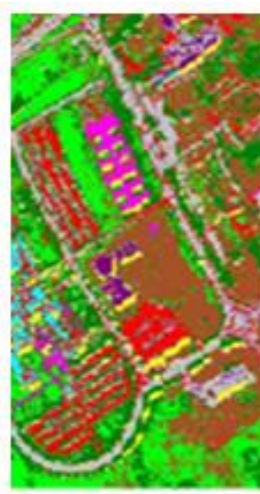

$60^{\circ}$

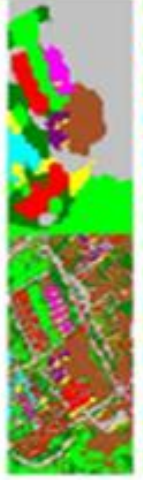

(b)

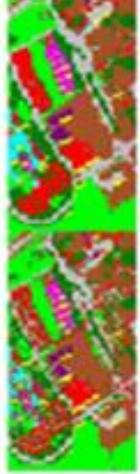

(2)

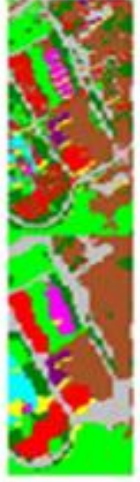

(J)

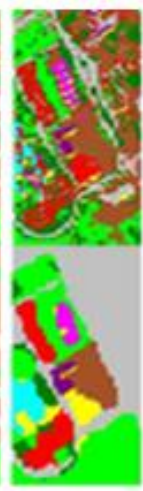

(c)
Fig. 3: Analysis for the free parameters $\beta$ and $\gamma$ for the University of Pavia image. (a) classification map obtained by the SVM method. In the top row of (b)-(e), $\gamma$ is fixed as 10-5. (b)-(e) Organization maps obtained by the proposed ERW method with (b) $\beta=100$, (c) $\beta=700$, (d) $\beta=103$, and (e) $\beta=104$. In the bottom row of (b)-(e), $\beta$ is fixed as 700. (b) -(e) Organization maps with (b) $\gamma=10-1$, (c) $\gamma=10-4$, (d) $\gamma=10-6$, and (e) $\gamma=10-7$.(e) Organization maps with (b) $\gamma=10-1$, (c) $\gamma=10-4$, (d) $\gamma=10-6$, and (e) $\gamma=10-7$

From all unearthly spatial characterization based calculations preferred execution over SVM in exactness In the order comes about got by the SVM, LORSAL, ERW, LORSAL-ERW. Both ERW and LORSAL ERW strategies to enhance better execution of pixel insightful characterization. In any case, the registering streamlining is additionally assessed utilizing MATLAB on the PC with 3.5ghzCPU and 8GBmemory.ERW based techniques is moderately quick with $1.9 \mathrm{sec}$ for preparing University of Pavia picture.

The Table 1 indicates OA, AA,KAPPA coefficients of various techniques, which are 3 broadly quality measurements for hyper otherworldly picture arrangement.

\begin{tabular}{|c|c|c|c|}
\hline Methods & $\begin{array}{c}\text { Table } 1 \\
\text { OA }\end{array}$ & AA & Карра \\
\hline SVM & 80.99 & 88.28 & 76.16 \\
\hline LORSAL & 80.11 & 87.70 & 75.09 \\
\hline SVMMRF-NE & 86.89 & 92.12 & 83.14 \\
\hline SVMMRF-E & 87.63 & 93.41 & 84.07 \\
\hline LORSAL-MLL & 85.57 & 92.54 & 81.80 \\
\hline MPM-LBP & 85.78 & 92.20 & 82.05 \\
\hline EPF & 87.83 & 89.24 & 84.26 \\
\hline ERW & 95.97 & 95.06 & 94.56 \\
\hline LORSAL-ERW & 96.60 & 96.28 & 95.40 \\
\hline
\end{tabular}

\section{Experiments with the Salinas Data Sets}

In this analysis performed on Salian picture in this picture contains 16 distinct classes characterization comes about acquire by various strategies. The proposed ERW demonstrates best execution as far as OA (98\%), AA(96\%),KAPPA(97\%).ERW technique best execution as far as precision especially when number examples are restricted.

\section{E. Indian Pines Data sets}

In this trial performed on INDIAN PINES picture. the beneath figure 7 indicates order comes about acquired by various strategies. The preparation tests are $10 \%$ of 10249 reference tests. In the figure commotion is expelled of SVM grouping map by the ERW technique.
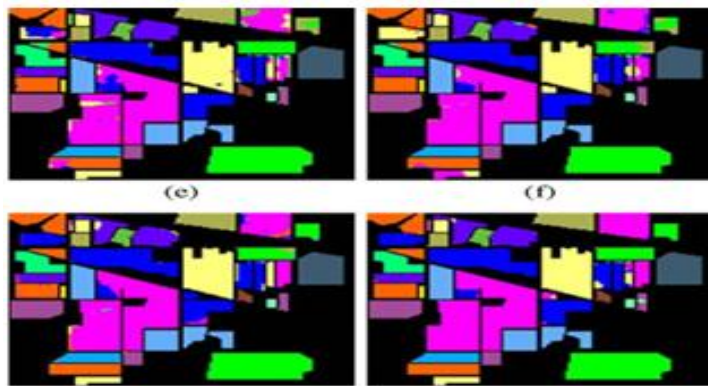

Fig. 5: Arrangement comes about (Indian Pines picture) got by (a) SVM strategy, (b) LORSAL technique, (c) EMP strategy, (d) LORSAL-MLL strategy, (e) MPM-LBP strategy, (f) EPF technique, (g) IFRF strategy, and (h) ERW method. Numbers in the brackets allude to the general characterization accuracies given in percent. (a) SVM (81.3). (b) LORSAL (81.7) (c) EMP (93.6). (d) LORSAL-MLL (92.1). (e) MPM-LBP (93.1). (f) EPF (93.2). (g) IFRF (96.4). (h) ERW (98.7).

The below table 2 shows OA, AA,KAPPA results and better performance result is ERW method.

Table 2

\begin{tabular}{|c|c|c|c|c|c|c|c|c|c|c|}
\hline \multirow{2}{*}{ Matlows } & \multirow{2}{*}{ Menis } & \multicolumn{9}{|c|}{ thenges } \\
\hline & & T5 & 8 & 36 & $\%$ & 16 & 15 & $3 n$ & 35 & W \\
\hline \multirow{3}{*}{ 5VY } & of & 52865 & 618025 & निख्याओ & $77 \times 15$ & $\operatorname{ses} 801 .+1$ & 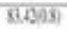 & 855508) & 8.201 & 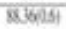 \\
\hline & H & $52 \mathrm{mad}$ & $58 \times 22)$ & 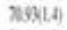 & $76651.8)$ & 7ธ8:15 & вะखนі & MES(1) & 859968 & (6\%)12) \\
\hline & Kipe & finges) & 506339 & $x \in(13)$ & 7421.5? & x.1s159 & 605(3) & saros) & scabs & \$COD) \\
\hline \multirow{3}{*}{ LoRSL. } & की & $50+201$ & $(655+2)$ & 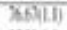 & त्र.25(1) & 81855 & 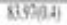 & 56:505 & 8.1.1.6. & SNand \\
\hline & M & $63(20)$ & noat3 & 853819 & stap?) & 8.17.4 & 85ibat) & nemor) & 912410 & Keol12) \\
\hline & Kopa & 596038 & 02102.1 & $735012 ?$ & $x \sin (3)$ & 7).xas? & s17ad: & 83505) & $85.126+1)$ & $85724)$ \\
\hline \multirow{3}{*}{$\mathrm{EQ}$} & of & क्जिय) & 8.11153 & 8315 & श्र०508) & 923645 & $93 \times 55$ & कinod & $860 \times 39$ & (86012) \\
\hline & AH & $62 \times 3.1)$ & 74542. & $862+15)$ & 825)(15) & $9006013 \%$ & s.senis & 935ting) & M34(1) & 56393) \\
\hline & $\mathrm{K}=\mathrm{mo}$ & 9.95015) & 700036 & (5.9.9.1.7) & ตnอง & 912nish & 929006 & MU:(0) & 98Mots & 6.at2) \\
\hline \multirow{3}{*}{ LesL-MeL } & of & $60601)$ & XaA, & 81:25) & 9..15(1) & 951013 & $946607)$ & क्र०⿴囗十 & 655019 & 912:29 \\
\hline & A & xo605: & seren & 9.5309) & و\$9t0) & 965329 & 97MDE & ทาג195) & 98906 & 5.1928 \\
\hline & Kipp & Q Clls5) & 73030 & 865925 & ness:-1) & Q2.1001. & 9.35:5: & s5er(10) & senten & 56.7201 \\
\hline \multirow{3}{*}{ NENLB? } & 04 & 60. I655) & $72 \times 15$ & हब(शाग) & 9x/312 & 931510 & प्रक्जी & क्रav0. & $973 \times 1$ & 9ग7रम \\
\hline & A & $72 \times 393$ & 81.51.3) & 9.5005) & 44sese & 90005 & 9.005: & กาว & $x: \times 10$ & 503027 \\
\hline & $x_{2}$ & S(11) 5.7$)$ & 685313 & selvals) & $9.48(A)$ & 213บ) & 中⿰冫广) & s090. & 968409 & 924:5) \\
\hline \multirow{3}{*}{ E } & of & 6055 & 785215) & (8240) & कृष्S(1) & क्राक & फ22067 & क6. & 9:5015 & 90005 \\
\hline & A & $6(12+2$ & $\times 2633$ & x:ssi.1) & 923410 & sulas & 2.9.65) & 958808 ; & genes & 98025 ? \\
\hline & Kype & Goos 5: & $70 \times($ a) & Dossan & 91. & 986013i & Y.1929) & 855013! & $96 \times 108$ & 9146029) \\
\hline \multirow{3}{*}{ HWF } & 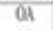 & $72+63.11$ & $81 \times 22)$ & श्रुपा. & क्र. $1 \times 05$ & 933519 & प्र(56) & 82502 & $5 \times 8003$ & Q. $1+23$ \\
\hline & At & 8126 & N.93:9 & seas.t. & भSसाम & 965113 & S:13:26: & resed) & xostes & 54605 \\
\hline & Kappe & (201333) & ser.25) & 22ख16 & 8AD05) & 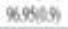 & 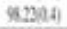 & ravo: & 986) & novali \\
\hline \multirow{3}{*}{$E \times W$} & of & $x 3609$ & 80021 & $50 \leq 13)$ & n? 3406 & s.tall & 58920) & Y01502) & nelest & read \\
\hline & $\mathrm{AA}$ & secus & scos: & \$SLIS:. & \%.361.7) & *6s:L1) & 5x.1As? & 28.9519 & 57819 & S.ISAS \\
\hline & Kapa & 7GSLD) & $8 \times 129$ & 85,3015) & nina? & 8.3uts? & 587+2) & Mato: & manes & 9.9.2) \\
\hline
\end{tabular}

\section{Conclusion}

In ghastly spatial arrangement of the picture is performed on ERW strategy. The exactness of SVM can be Enhanced by utilizing ERW compared with different characterization of unearthly spatial strategies, the favorable position is precision if the number of typing tests extremely smaller value in genuine application because of high precision and low computational burden. The EXTESION of this venture is K-MEANS utilized rather than PCA,t o enhance the exactness.

\section{References}

[1] A.Vlla,J, A.Benediktsson, J.Chanussot, and C.JUTTEN, Hyper spectral image classification with independent component discriminative analysis.IEEE Trans.Geosci.Remote sens, vol .49.no. 12 ,pp 4865-4876 dec.2011.

[2] B.Demir and S. erturk Empiricial mode decomposition of hyper spectral images for support vector machine.IEEE trans geosci remote sens vol 48 no. 11 pp4071-4084,nov 2010

[3] SK Hasane Ahammad and Rajesh Image Processing Based Segmentation Techniques For Spinal Cord In MRI, Indian Journal Of Public Health Research \& Development, June 2018, Vol. 9, No. 6

[4] M.Pedergnana p.Marpu. m.mura J.A Benediktsson and L.BRUZZONE Anovel tech. for optimal fearure selection in attribute profile based on genetic algorithms IEEEtrans Geosci remote sens vol.51.no 6 pp 35143528 jun 2013. 